\section{Gene expression tryptophan aspartate coat protein in determining latent tubercu- losis infection using immunocy- tochemistry and real time polimerase chain reaction}

Rebekah J. Setiabudi, ${ }^{1}$ Ni Made Mertaniasih, ${ }^{1}$ Muhammad Amin, ${ }^{2}$ Wayan Tunas Artama ${ }^{3}$

1,2Department of Medical Microbiology, TB Laboratory Institute of Tropical Disease; ${ }^{3}$ Department of Pulmonology, Faculty of Medicine, Universitas Airlangga; ${ }^{4}$ Faculty of Veterinary, Gajah Mada University, Indonesia

\section{Abstract \\ Background: Tuberculosis (TB)} remains a major cause of morbidity and mortality worldwide. Problem of Latent Tuberculosis Infection (LTBI) is increasing in number especially in countries with high TB incidence rate, such as Indonesia. Although not every LTBI will become active $T B$, if untreated and not handled appropriately it can still be a source of transmission and may increase the rate of resistance to the first-line TB drugs. Mycobacterium tuberculosis as a cause of tuberculosis disease is an intracellular pathogens that survives within the phagosome of host macrophages. Several host factors are involved in this process, including the Tryptophan Aspartate-containing Coat Protein (TACO). TACO is a protein recruited and retained by viable Mycobacterium tuberculosis on the surface of the phagosome membrane to maintain its survival in phagosome, because the presence of TACO plays an important role in inhibiting the fusion of phagosomes and lysosomes.

Objective: the aim of this studyis to assess the difference of gene expression TACO protein in Latent Tuberculosis Infection (LTBI) and healthy people.

Method: A preliminary studyof mRNA examination of TACO protein using Immunocytochemistry (ICC) and Real Time-Polimerase Chain Reaction (RT-PCR) method by a PCR Light Cycler 2.0 machine (Roche) in LTBI and healthy groups.

Results: 18 samples of peripheral blood monocyte cells (PBMCs) were collected and divided into 2 groups. We found that there was a significantly difference between the 2 groups of samples.
Conclusion: Further research is required to consider that the measurement of TACO expression using RT-PCRcan used as one of the other method to determine LTBI.

\section{Introduction}

Tuberculosis (TB) remains a major cause of morbidity and mortality worldwide. Currently reported, one-third of the entire world's population has been infected by Mycobacterium tuberculosis, the cause of tuberculosis. By 2015 it is estimated that there are 10.4 million people worldwide suffering from clinical TB, 5.9 million $(56 \%)$ are male, 3.5 million (34\%) females and 1.2 million $(11 \%)$ children. There are an estimated 1.4 million deaths due to TB by 2015 and an additional 0.4 million deaths due to TB among people suffering from HIV infection. ${ }^{1}$ TB was one of the top 10 causes of death worldwide in 2015 , and TB has killed more individuals every year than any other pathogenic germ. In 2016 WHO report, Indonesia is the third largest countries for number of TB cases after India.

In the United States, it is estimated more than 11 million people are asymptomatically infected by Mycobacterium tuberculosis (Latent Tuberculosis Infection, LTBI), estimated at $4 \%$ of the total population. Although not every LTBI will become active TB, approximately $5-10 \%$ will become active, which is equivalent to an estimated 550,000 to $1,100,000$ LTBI to active TB at some point in life, except the population suspect LTBI is receiving adequate therapy for LTBI. ${ }^{2}$ If LTBI is not properly diagnosed and not given appropriate treatment, LTBI may also be a source of infection later, and inappropriate treatment of LTBI may increase the rate of resistance to the first-line TB drugs. So, proper identification of LTBI and therapy in people at high risk for TB can be the basis for the determination of LTBI treatment with the aim of early detection and prompt treatment.

Mycobacterium tuberculosis has been known to have the ability to develop several mechanisms to avoid the primary host cell defense that attack them through phagocytosis by macrophages. ${ }^{3}$ The introduction of Mycobacterium tuberculosis by macrophages and dendritic cells occurs through the identification of cell wall antigens present on the surface of Mycobacterial cells. ${ }^{4}$ This recognition occurs through various receptors present in the macrophages, including mannose receptors, scavenger receptors and complement
Correspondence: Rebekah J. Setiabudi, Department of Medical Microbiology, Faculty of Medicine and TB Laboratory Institute of Tropical Disease, Universitas Airlangga. Tel.: +628123031557

E-mail: rebekahsetiabudi@gmail.com.

Key words: tryptophan aspartate containing coat protein, immunocytochemistry, real timepolimerase chain reaction, Mycobacterium tuberculosis, latent tuberculosis infection.

Contributions: All the authors contributed equally.

Conflict of interest: The authors declare no potential conflict of interest

Acknowledgements: The authors thank to director and staff of Dr. Soetomo General Hospital Surabaya Indonesia, Pegirian Primary Health Care, Institute of Tropical Diseases Airlangga University and Airlangga University Hospital.

Conference presentation: The paper had been presented at INSBIOMM International conference on latest perspectives on Infectious Diseases, including Biothreats and Military Medicine, in August 27-28, 2019, Surabaya, Indonesia.

Received for publication: 17 February 2020. Accepted for publication: 1 July 2020 .

This work is licensed under a Creative Commons Attribution-NonCommercial 4.0 International License (CC BY-NC 4.0).

(C) Copyright: the Author(s), 2020

Licensee PAGEPress, Italy

Infectious Disease Reports 2020; 12(s1):8733 doi:10.4081/idr.2020.8733

receptors. ${ }^{5}$ It is said that the pathogenic Mycobacterium tuberculosis enters the macrophage cells through the mannose receptors. Binding of Mycobacterium tuberculosis with mannose receptors in this macrophage facilitates the survival of these microorganisms in macrophage cells. After entering into macrophage cells through the process of phagocytosis, Mycobacterium tuberculosis gets trapped in a room formed by the plasma membrane of macrophage cells called phagosomes while awaiting further degradation. ${ }^{4,6}$ It is in this phagosome that Mycobacterium tuberculosis exhibits various self-defense mechanisms so that the degradation process does not continue and Mycobacterium tuberculosis can survive and multiply in macrophage cells. The biomolecular processes occurring within macrophage cells and how they can cause Mycobacterium tuberculosis to survive in macrophage cells, are still continuously 
investigated.

Tryptophan Aspartate-containing Coat protein (TACO) is recruited and retained on the surface of the phagosome membrane by viable Mycobacterium tuberculosis to assure its survival in macrophage cells. ${ }^{7}$ Previous research suggests that a phagosome containing dead Mycobacterium bovis will quickly be eliminated from macrophages, whereas phagosomes that contain viable Mycobacterium bovis will be more stable in macrophages because of their ability to maintain TACO. ${ }^{8,9}$ Mycobacterium bovis bacteria used in the BCG vaccine, in the form of an attenuated germ, will be completely digested and eliminated in Kupffer cells as macrophage cells in hepatic organ tissue, whereas its TACO expression is considerably less. Phagosomes containing less than 5 Mycobacterium tuberculosis bacteria can not maintain TACO while phagosomes containing more than 5 bacteria clearly retain TACO on the phagosome membrane. ${ }^{8}$ Other studies have indicated that the human promonocytic cell line THP-1 which infected by live and heat-killed Mycobacterium lepraeexhibited that viable Mycobacterium leprae utilizes unknown strategy to suppress innate immune activities and maintain TACO expression. ${ }^{10}$ Anand et al. said that the suppression of TACO gene expression both before and after infection will support the survival of Mycobacterium tuberculosis in macrophage cells. ${ }^{11,12}$ Based on the study before, this research was conducted for to know the differences of gene expression of TACO protein in LTBI and healthy people.

\section{Materials and Methods}

This research was conducted from February to October 2015. 18 samples were peripheral blood monocyte cells (PBMCs) from active TB patient's close contact family at Dr. Soetomo General Hospital Surabaya Indonesia and Pegirian Primary Health Care and healthy people who have never experienced symptoms of tuberculosis; they were aged 21 to 65 years, not suffering from diabetes mellitus, HIV infection and not smoking. Informed consent was given for laboratory examination. This study was approved by the Ethical Committee in Health Research of Dr. Soetomo General Hospital, Surabaya, Indonesia (no. 404/Panke.KKE/VIII/2015).

Whole blood samples were examined in the TB Laboratory, Department of Clinical Microbiology, Dr. Soetomo General Hospital, with standard culture method. The standard culture method BACTEC MGIT 960 system (BD) liquid medium and
Lowenstein-Jensen solid medium were used for detection and identification of mycobacteria. The serology testing of Interferon Gamma Releasing Assays (IGRAs) using Interferon Gamma Releasing Assays-Gold In Tube test (IGRA-GIT) was done at the Institute of Tropical Diseases, Airlangga University, and it was used to separate LTBI from healthy people. Immunocytochemistry (ICC) Examinations were done in Electron Microscopy Unit and Intergrated Laboratory Airlangga University with monoclonal antibody bought from MyBioSource, Inc., USA, and it was used to determine the existence of TACO proteins on the surface of PBMCs.

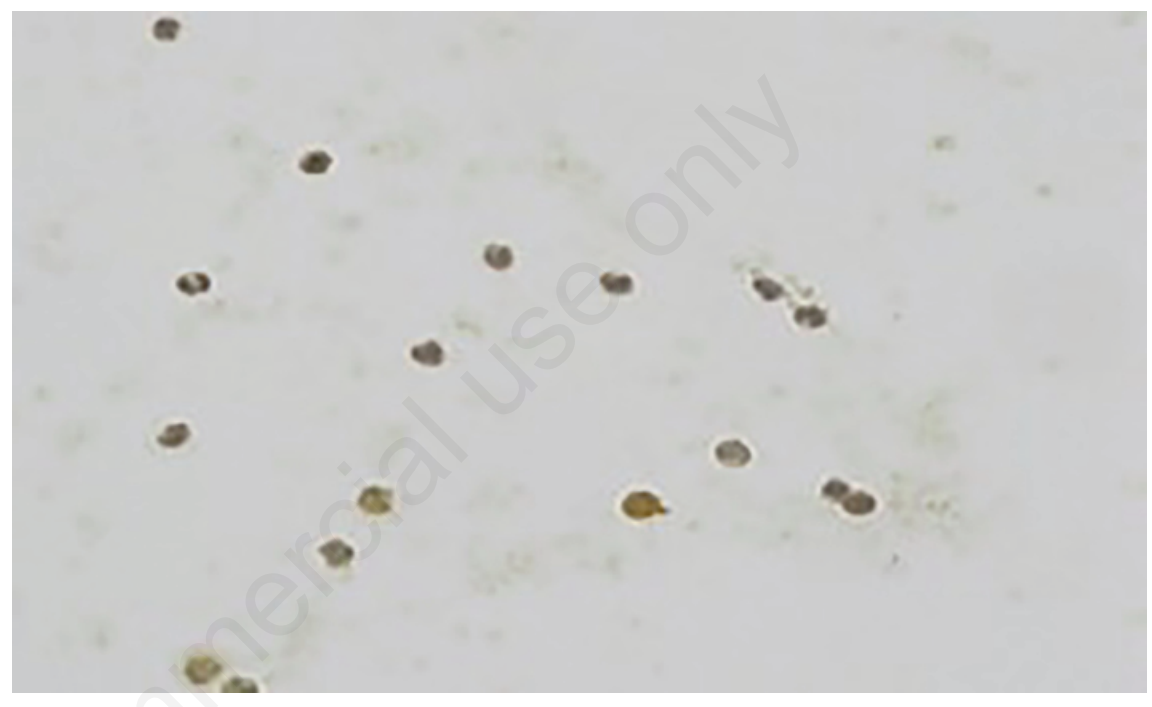

Figure 1. ICC's result of LTBI patient (Chromogen and Mayer's hematoxylin, 400x). Several PBMCs with TACO protein were detected.

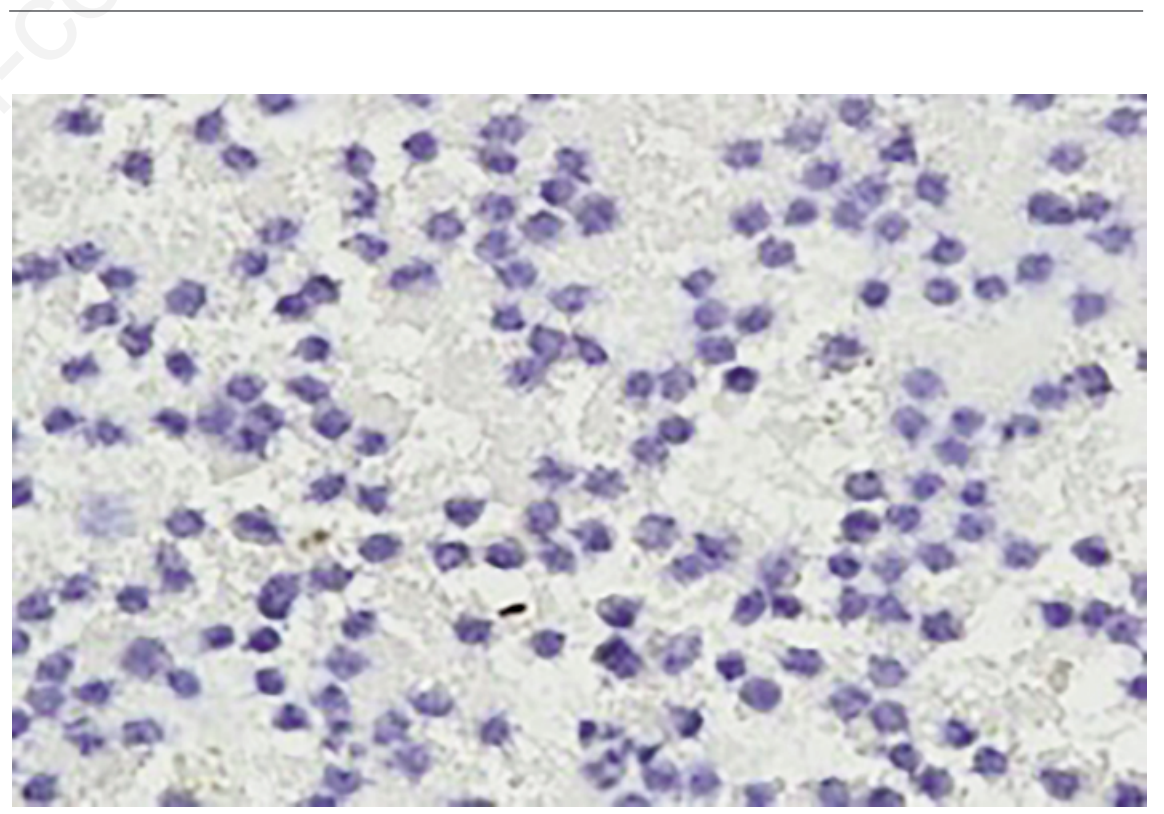

Figure 2. ICC's result of healthy people (Chromogen and Mayer's hematoxylin, 400x). Many PBMCs without TACO protein were detected.
The Real Time PCR using LightCycler machine 2.0 (Roche) was done in Airlangga University Hospital. PBMCs isolation from whole blood specimens was used using a Ficoll-Histopaque 1077 solution, a suitable reference procedure to obtain pure mononuclear cells. ${ }^{13}$ The RNA extraction was done using Geneaid for Blood and Tissue Kit then measured the number of RNAs eligible for RT-PCR using Nano Drop. The TACO gene was amplified using the primers 5'CCAGTGCTATGAGGATGTGCGCG and 5'-GACACGACTCGCTTGTCA-AGGC-3 '. These genes were amplified using PCR programs: $94^{\circ} \mathrm{C}$ for 45 cycles; $60^{\circ} \mathrm{C}$ for 45 cycles; $72^{\circ} \mathrm{C}$ for 90 cycles $\left(22\right.$ cycles). ${ }^{11}$ 


\section{Results}

From the statistical analysis using Fisher exact (Table 1) there was no significantly difference from TACO expression by Immunocytochemistry (ICC) in latent tuberculosis infection (LTBI) patients with healthy people.

From the statistical analysis using $\mathrm{T}$ test for 2 free samples (Table 2), we then obtained the following results:

There was a significantly difference from TACO expression by RT-PCR in latent tuberculosis infection (LTBI) patients with healthy people.

In patients with latent tuberculosis infection (LTBI), the TACO expression rate is higher than in healthy people.

\section{Discussion}

From the results of the ICC (Figures 1 and 2), it appears that there are differences in the number of healthy cells that do not express TACO protein between healthy people and LTBI patients. In LTBI patients, it was seen that a smaller number of cells did not express TACO protein; in other words, most of the cells appeared to express TACO protein. This evidence showed that the existence of self-defense mechanism carried out by Mycobacterium tuberculosis in cells to maintain their life by forming TACO proteins on the surface of PBMCs to inhibit the fagosome and lysosome fusion that can kill the bacteria. ${ }^{14}$

By mRNA examination of TACO proteins using RT-PCR, statistical significantly differences were apparent between LTBI patients and healthy people. It was also found that TACO protein expression was higher in patients with latent tuberculosis infection than in healthy individuals. This is consistent with the theory that in LTBI patients whose monocyte cells have been exposed to Mycobacterium tuberculosis bacteria will inevitably show a higher expression of TACO protein than healthy people whose cells have not been exposed to the bacteria. Patients with latent tuberculosis infection (LTBI) manage to limit infection through various mechanisms. ${ }^{14,15}$ First time that plays a role is the natural immunity, the phagocyte cells, among others, monocytes and neutrophils will migrate to the site of infection, but they are unable to kill the bacteria efficiently. ${ }^{16,17}$ After phagocytosis process of Mycobacterium tuberculosis, the phagocyte cells induce Interferon Gamma (IFN- $\gamma$ ) production which will stimulate the increase of Reactive Oxygen Species (ROS) in the host cell as an antimicrobacterial factor. ${ }^{18}$ On the other hand, IFN- $\gamma$ as a proinflammatory cytokine will be excluded to activate T lymphocytes, as an adaptive immune defence. IFN-r will also activate inducible Nitric Oxide synthase (iNOS) to produce Nitric Oxide (NO), which then reacts with radical oxygen to form Reactive Nitrogen Intermediate (RNI). NO and RNI play an important role as antimicrobacterials too. In addition to IFN-r, phagocyte cells also secrete Toll Like Receptor 2 (TLR2) which induces the release of Nuclear Factor Kappa $\mathrm{B}(\mathrm{NF} \kappa \mathrm{B})$ which is a protein complex responsible for controlling DNA transcription, the production of other cytokines and the survival of phagocyte cells. ${ }^{10}$ Furthermore, phagocyte cells also secrete other proinflammatory cytokines as intercellular communication signals that will work with other immunocompetent cells to eliminate Mycobacterium tuberculosis infection. ROS is also produced by various immunocompetent cells, including macrophages, when in contact with bacteria or other foreign bodies. Excessive ROS production will be regulated by enzymes such

Table 1. Results of immunocytochemistry in latent tuberculosis infection (LTBI) patients and healthy people.

\begin{tabular}{lcccc} 
Group & N & Negative results & Positive results & P-value \\
LTBI & 9 & $6(66,7 \%)$ & $3(33,3 \%)$ & 0,500 \\
Healthy people & 9 & $7(77,8 \%)$ & $2(22,2 \%)$ & \\
\hline
\end{tabular}

Table 2. Results of RT-PCR TACO protein expression in latent tuberculosis infection (LTBI) patients and healthy people.

\begin{tabular}{lccc} 
Group & N & Average \pm Standard Deviation & Value p \\
LTBI & 9 & $1,002 \pm 0,070$ & $<0,0001$ \\
Healthy people & 9 & $0,681 \pm 0,083$ & \\
\hline
\end{tabular}

as catalase, superoxide dismutase (SOD), glutathione peroxidase $(\mathrm{GPx})$ and glutathione reductase (GR) in homeostatic processes. ${ }^{19}$

During this time, the bacilli resist the bactericidal mechanisms of the macrophage (phagolysosome) by preventing phagosome-lysosome fusion, survive and multiply in the phagosome, and cause macrophage necrosis. ${ }^{20,21}$ Some surviving and live bacteria can express TACO protein in order to escape its destructive process through the fusion of phagosomes with lysosomes. ${ }^{22}$ This is also consistent with the theory that TACO protein is recruited and retained on the surface of the phagosomal membrane depending on the bacterial present in the phagosome. As a self-defense mechanism of the bacterium Mycobacterium tuberculosis, retention of TACO aims to avoid fusion of phagosomes with lysosomes so that bacteria do not experience bactericidal effects through destruction by the proteolytic enzymes present in the lysosomes. ${ }^{22}$ TACO prevents the fusion of phagosomes by lysosomes either by modulating actin cytoskeleton. ${ }^{23}$ or by setting the calcium-dependent signaling process, but in further analysis it was found that there is a more important role of TACO in the mediation of $\mathrm{Ca}^{2+}$ ion influx through interaction with PLC- $y 1$ and activation of calcium dependent phosphatase calcineurin which is worked in avoiding the fusion of phagosomes with lysosomes. So the absence of TACO will cause the failure of this process and the absence of calcineurin activation. ${ }^{24}$ This may also be through the process of dephosphorylation of substrates released by host cells that are still unknown. This process facilitates the survival of Mycobacterium tuberculosis in the phagosome for a long time. ${ }^{7,25}$ In addition to the inhibition of fusion of phagolisosomes, it turns out that TACO protein also plays a role in cooperation with other proinflammatory cytokines that suppress NF- $\kappa \beta$ activation mediated by TLR-2; TLR-3, TLR-4 and TNF- $\alpha$ stimulated by NF- $\kappa \beta$ activation and activation of IFN- $\beta$ promoter. ${ }^{26}$ One possible explanation is that TACO proteins are directly related to molecules that decrease both TLR and TNF- $\alpha$ signaling, such as NIK (NF- $\kappa \beta$ inducing kinase, inhibitor (1) $\kappa \beta$ or NF- $\kappa \beta$.) Another possibility is the TACO protein associated with molecules such as nicotinamide adenine dinucleotide phosphate (NADPH) oxidase to inhibit TLR-mediated signaling, so it can be seen here that TACO proteins also play a role in reducing signaling pathways leading to activation of natural immunity. ${ }^{10,27}$

LTBI: latent tuberculosis infection. 


\section{Conclusions}

Based on the preliminary study results above, further research is required to consider that the measurement of mRNA of TACO expression using RT-PCRcan be used as one of the other method to determine LTBI, i.e. Interferon Gamma Releasing Assay (IGRA test) and culture.

\section{References}

1. World Health Organization. 2016. Global Tuberculosis Report.

2. CDC. Latent tuberculosis infection :a guide for primary health careproviders; 2013. Available from: www.cdc.gov/tb/publications/ltbi/diagn . osis.htm.

3. Smith I. Mycobacterium tuberculosis pathogenesis and molecular determinants of virulence, Clin Microbiol Rev 2003;16:463-496.

4. Raja A. Immunology of Tuberculosis. Indian J Med Res 2004 120:213-232.

5. Kang PB, Azad AK, Torrelles JB, et al. 2005. The human macrophage mannose receptor directs Mycobacterium tuberculosis lipoarabinomannan-mediated phagosome biogenesis. J Exp Med 2005;202:987 - 999 .

6. Adugna W, Tessema TS, Keskes S. An insight review on Immunopathogenesis of bovine and human mycobacteria infections. An insight review on Immunopathogenesis of bovine and human mycobacteria infections. Int $\mathrm{J}$ Med Med Sci 2013; 6:42-52.

7. Pieters J, Muller P, Jayachandran R. On guard: coronin proteins in innate and adaptive immunity. Nat Rev Immunol 2013;13:510-518.

8. Ferrari G, Langen H, Naito M, Pieters J. 1999. A coat protein on phagosomes involved in the intracellular survival of mycobacteria. Cell 1999;97:435-447.

9. Schuller S, Neefjes J, Ottenhoff T, et al.
Coronin is involve in uptake of Mycobacterium bovis BCG in human Macrophages but not in phagosome maintenance. Cell Microbiol 2001;3:785-793.

10. Tanigawa K, Suzuki K, Kimura H, et al. Tryptophan aspartate-containing coat protein (CORO1A) suppresss Toll-like receptor signaling in Mycobacterium leprae infection. Clin and Exp Immunol 2009: 156:495-501.

11. Anand PK, Kaul D. Downregulation of TACO gene transcription restricts mycobacterial entry/survival within human macrophages. FEMS Microbiol Lett 2005;250:137-144.

12. Anand PK, Kaul D, Sharma M. Green tea polyphenol inhibits Mycobacterium tuberculosis survival within human macrophages. Int J Biochem Cell Biol 2006;38:600-9.

13. Holtz A, Toll AD, Susan W, et al., Establishing the Purity of Mononuclear Cell Preparations Using Morphology and Flow Cytometry. Department of Biology, Drexel University, Philadelphia. Departement of Pathology, Thomas Jefferson University Hospital, Philadelphia. Poster Presentation; 2009.

14. Ahmad S. Pathogenesis, immunology, and diagnosis of latent Mycobacterium tuberculosis infection. Clin Dev Immunol 2011;814943.

15. Chee CB, KhinMar KW, Gan SH, et al. Latent tuberculosis infection treatment and $\mathrm{T}$ cell responses to Mycobacterium tuberculosis specific antigens. Am J Respir Crit Care Med 2007;175:282-7.

16. Tsolaki AG. 2009. Innate immune recognition in tuberculosis infection. Advancesin Exp Biol Med 2009;653:185-197.

17. Aziz MA, Tsolaki AG. 2013. Chapter 4 Mycobacterium tuberculosis: Innate Immune Recognition and Persistence (C) 2013 Copyright Landes Bioscience and Springer. 63-81.
18. Herbst S, Schaible UE, Schneider BE. Interferon gamma activated machophages kill mycobacteria by nitric oxide induced apoptosis. PLoSOne 2011:6:e19105.

19. Hor-Yue Tan, Ning Wang, Sha Li, et al. The Reactive Oxygen Species in Macrophage Polarization: Reflecting Its Dual Role in Progression and Treatment of Human Disease. Oxid Med Cell Longev 2016:2795090

20. Vergne I, Chua J, Hwang-Ho Lee, et al. Mechanism of phagolysosome biogenesis block by viable Mycobacterium tuberculosis. PNAS 2005;102:40334038.

21. Deretic V, Singh S, Master S, et al. Mycobacterium tuberculosis inhibition of phagolysosome biogenesis and autophagy as a host defence mechanism. Cell Microbiol 2006;8:719-727.

22. Meena LS, Rajni. 2010. Survival mechanisms of pathogenic Mycobacterium tuberculosis H37Rv. FEBS J 2010;277: 2416-2427.

23. Jayachandran R, Sundaramurthy V, Combaluzier B, et al. 2007. Survival of Mycobacteria in Macrophages is Mediated by Coronin 1-Dependent Activation of Calcineurin. Cell 2007;130: 37-50.

24. Punwani D, Pelz B, Yu J, et al. Coronin1A : Immune Deficiency in Humans and Mice. J Clin Immunol 2015;35: 100-107.

25. Pieters J. Mycobacterium tuberculosis and the macrophage: maintaining a balance. Cell Host and Microbe 2008;3:399-407.

26. Scherr N, Jayachandran R, Mueller P, Pieters J. Interference of Mycobacterium tuberculosis with macrophage responses, Indian J of Exp Biol 2009;47:401-406.

27. Kaufmann SHE. 2001. How can Immunology contribute to the control oftuberculosis? Nat Rev Immunol 2001;1:20-30. 